\title{
Quantum Calculations of the Electronic Structure of a Many-Atom Plasma
}

\author{
S. M. Younger, A. K. Harrison, K. Fujima, ${ }^{(a)}$ and D. Griswold \\ Lawrence Livermore National Laboratory, Livermore, California 94550
}

(Received 23 November 1987)

\begin{abstract}
We have performed a set of prototypical quantum calculations of the electronic structure of a nineatom helium plasma over wide ranges of temperature and density. These calculations reveal the presence of very tightly bound quasimolecular states in high-density plasmas, even at temperatures high enough to ionize fully the component atoms. The results of the study suggest that there are four regimes in a plasma, dependent upon the relationship of the electron wavelength to the interionic spacing: lowdensity atomic, intermediate-density screened atomic, high-density quasimolecular, and very-highdensity homogeneous.
\end{abstract}

PACS numbers: $52.25 . \mathrm{Jm}, 31.20 . \mathrm{Sy}$

A thorough understanding of the structure and dynamics of high-energy-density plasma is crucial to the achievement of inertial confinement fusion, as well as to the modeling of dense astrophysical objects. By a highenergy-density plasma we mean plasma that is hot enough that significant ionization is expected to occur and dense enough that there is appreciable overlap between the electronic charge distributions of neighboring atoms. Fujima, Watanabe, and Adachi ${ }^{1}$ have recently reported the results of a series of calculations on compressed matter at zero temperature, as have Collins and Merts $^{2}$ below $500 \mathrm{~K}$. In this paper we report the first systematic quantum-mechanical calculation of the electronic structure of a high-energy-density plasma which takes explicit account of the many-atom nature of the plasma electronic interactions. The results of the calculations suggest that the plasma state at high energy density be classified into four phases, depending on the relationship of the plasma electron wavelength to the interionic spacing.

The calculations reported here were performed with a finite-temperature $X \alpha$ Hartree-Fock-Slater molecular structure code ${ }^{2}$ written by one of us (K.F.) on the basis of the self-consistent charge approximation of Rosen et $a l .{ }^{3}$ Numerical atomic basis orbitals were solutions of the one-electron wave equation containing the spherically symmetric potential $V=V_{\mathrm{C}}+V_{\mathrm{ex}}+V_{w}$. Here $V_{\mathrm{C}}$ is the Coulomb potential due to the nucleus and the direct part of the interelectronic interaction. $V_{\mathrm{ex}}$ is the Slater exchange potential. $V_{w}$ is a finite square-well potential centered at the nucleus which is of sufficient depth to contain most of the charge density of the basis orbitals. The well radius was taken equal to the lattice spacing, $d$. The atomic basis orbitals were adjusted at each molecular structure iteration in order to better adapt the basis set to the molecular potential. We set $\alpha=0.7$ in our calculations. This yields an atomic $1 s$ orbital which is too weakly bound, but it is expected to approximate well the exchange term at higher electron densities. The electron population was allocated according to a Fermi distribu- tion. We use the convention that negative and positive energy states correspond to bound and continuum electronic states, respectively. The ion positions remained fixed throughout each calculation. In the Born-Oppenheimer approximation, the resulting "snapshot" of the plasma has valid electronic wave functions for the given set of ion positions, because of the much faster relaxation time of the electrons compared to the ions. The correct treatment of ion dynamics is not expected to change our qualitative observations below. As a realistic yet computationally tractable plasma configuration, we chose a nine-atom body-centered-cubic cluster of helium atoms, illustrated in Fig. 1. While the energy levels and occupation numbers of such a cluster will obviously differ from those of a large system, we are interested primarily in the general trends, and the nine-atom bcc arrangement represents a reasonable nearest-neighbor configuration

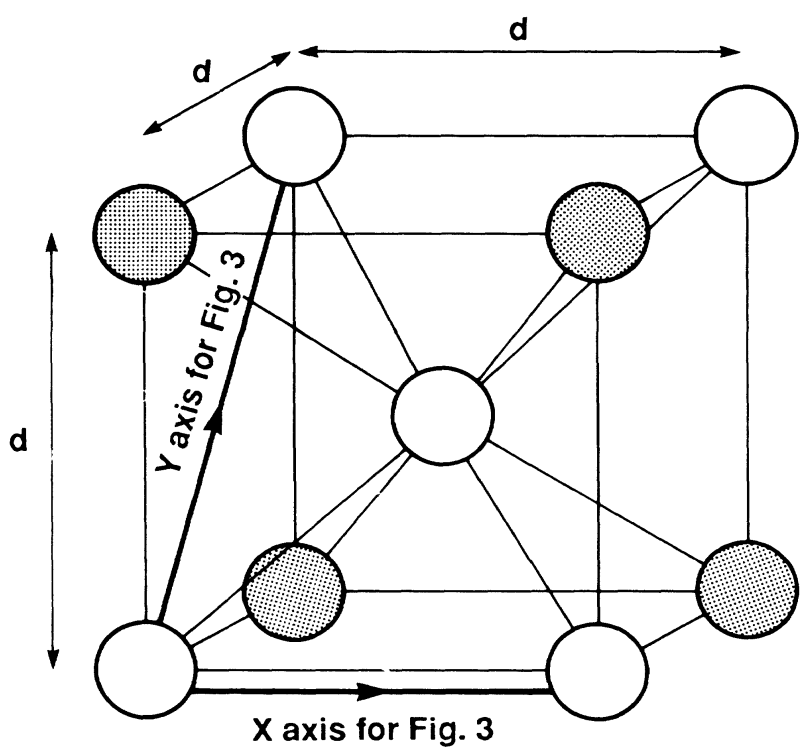

FIG. 1. Geometric configuration of the nine-atom bodycentered-cubic cluster. 
for study. We note that in very recent work $^{4}$ we have simulated the dynamics of clusters of up to 30 helium atoms in a Hartree-Fock approximation. In these calculations stochastic fluctuations led to the formation of temporary clusters of atoms which, although they lacked symmetry, possessed the same qualitative electronic structure features described below.

For most of the calculations we employed a basis set consisting of $1 s, 2 s, 2 p, 3 s, 3 p$, and $3 d$ orbitals. Selected calculations done with larger basis sets including $4 s, 4 p$, $4 d, 4 f, 5 s, 5 p, 5 d$, and $5 f$ orbitals did not differ significantly from the $1 s-3 d$ results.

We performed several series of calculations of the electronic structure of the helium cluster at different temperatures and plasma densities. First, a zero-temperature calculation was performed for a lattice spacing $d$ of $8 a_{0}\left(a_{0}=0.529 \times 10^{-8} \mathrm{~cm}\right)$. At this distance there is little interaction between the atoms. Next, the electron temperature was raised to $25 \mathrm{eV}$ and then to $50 \mathrm{eV}$. These values are significant in that they represent the first and second ionization potentials of the helium atom. As expected, the number of bound electrons on the cluster decreased with increasing temperature. Also, the energy of the lowest bound state decreased (became more negative), since there was less screening of the nuclear charge when the atom was partially ionized.

In the next phase of the calculations, we left the temperature fixed at $50 \mathrm{eV}$ and decreased the interionic spacing, i.e., increased the effective plasma density. Some of the results of these calculations are shown in Fig. 2. As the interionic spacing decreases from $8 a_{0}$ to about $2 a_{0}$, the eigenvalues, plotted in Fig. 2(a), become progressively less bound. This is because of the increased screening of the nuclear charge of the atoms due to the charge clouds of surrounding atoms. A noticeable change in the eigenvalues begins to appear at $4 a_{0}$, which is where the tails of the $1 s$ atomic bound orbitals just begin to overlap. The plasma is still mainly atomic in character event though there are significant perturbations due to neighbor-atom interactions.

A very different behavior begins to emerge at higher plasma densities. Between $2 a_{0}$ and $1 a_{0}$, there is an appreciable drop in the ground-state eigenvalue. It is remarkable that for $0.7 a_{0}<d<3 a_{0}$ this bound state is more tightly bound than any of the bound states associated with the isolated ions. Examination of the molecular orbitals associated with this state reveals that it is not atomic in character, but rather a hybrid, quasimolecular orbital the charge density of which is distributed among all of the nuclei in the cluster. Orbital hybridization occurs at high density where the interionic spacing is comparable to the wavelength of the bound electrons. The coincidence of a maximum in the atomic orbital and the location of a neighbor nucleus results in a strong sharing of electron density. In effect, the attractive potentials of several nuclei combine to create a deeper po- tential than that associated with any single atom. The bonding orbital that results is a chemical bond in the usual sense; what is remarkable is that it is occupied at temperatures large compared to the ionization energy of an isolated atom. Figure 2(b) shows that as the density increases the number of bound electrons on the cluster at first increases and then decreases. This is predicted by atomic theories of plasmas wherein at moderate densities recombination occurs to repopulate bound states and at
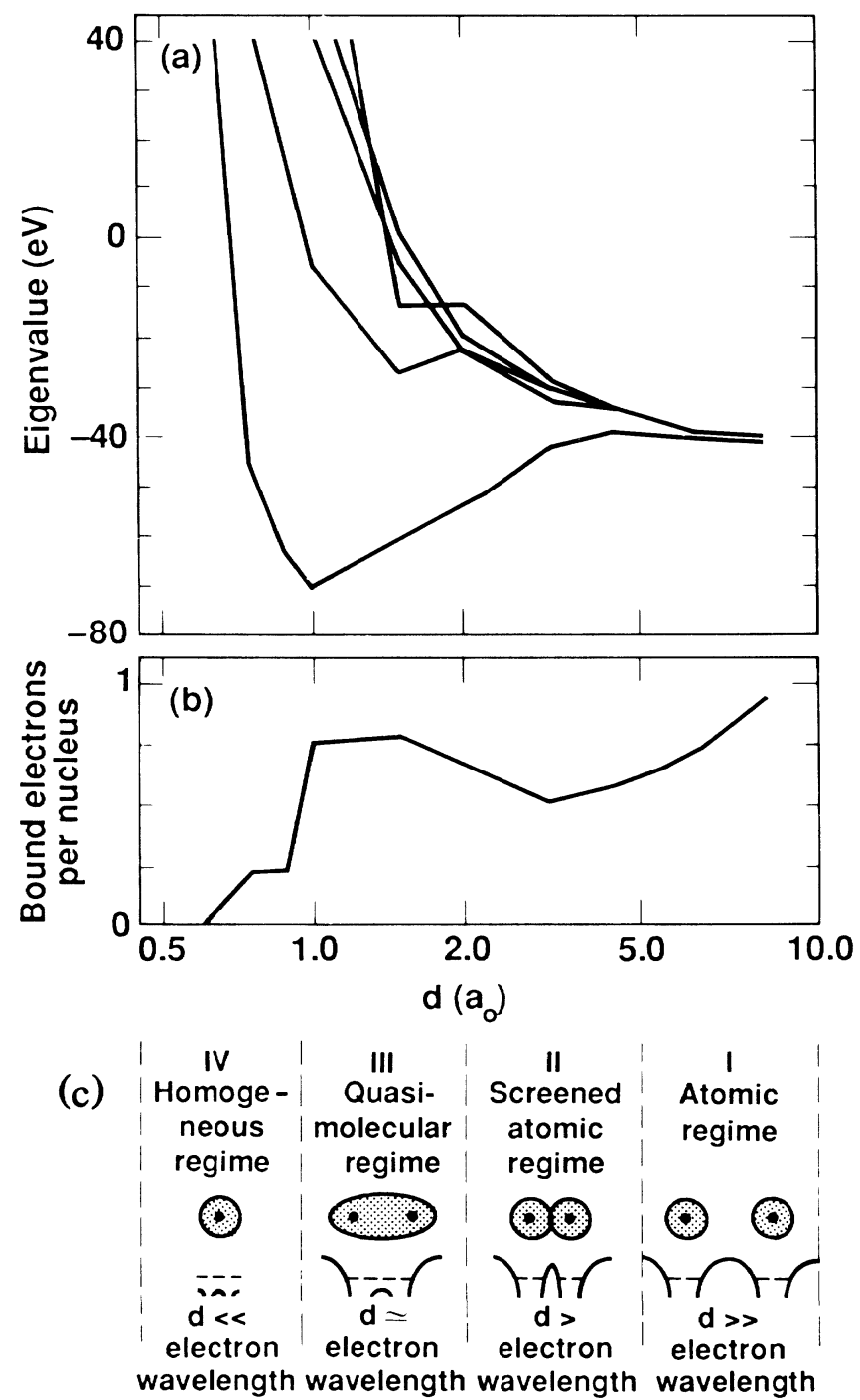

FIG. 2. (a) Eigenvalues of the nine-atom helium cluster which go to the $1 s$ state of atomic helium at large lattice spacing, plotted as a function of the lattice spacing. (b) Average number of bound electrons per atom of the cluster plotted as a function of lattice spacing for a plasma temperature of $50 \mathrm{eV}$. (c) Illustration of the four regimes of the helium plasma in terms of the atomic overlap, the structure of the potential curves determining the electronic motion, and the relationship of the interatomic distance to the characteristic wavelength of the electron. 
high densities pressure ionization occurs, reducing the bound-electron population.

In the third phase of our calculations, we left the lattice spacing fixed at $1 a_{0}$ and increased the electron temperature from 50 to $1000 \mathrm{eV}$. The ground-state energy of the cluster changed very little with temperature, although the number of bound electrons decreased mono- tonically to 0.1 at the highest temperature.

From the present calculations we suggest that there are four regimes of a high-energy-density plasma, all of which can be understood in terms of the relationship between the wavelength of the plasma electrons and the interionic separation. These regions are depicted schematically in Fig. 2(c) and illustrated in Fig. 3 by charge-
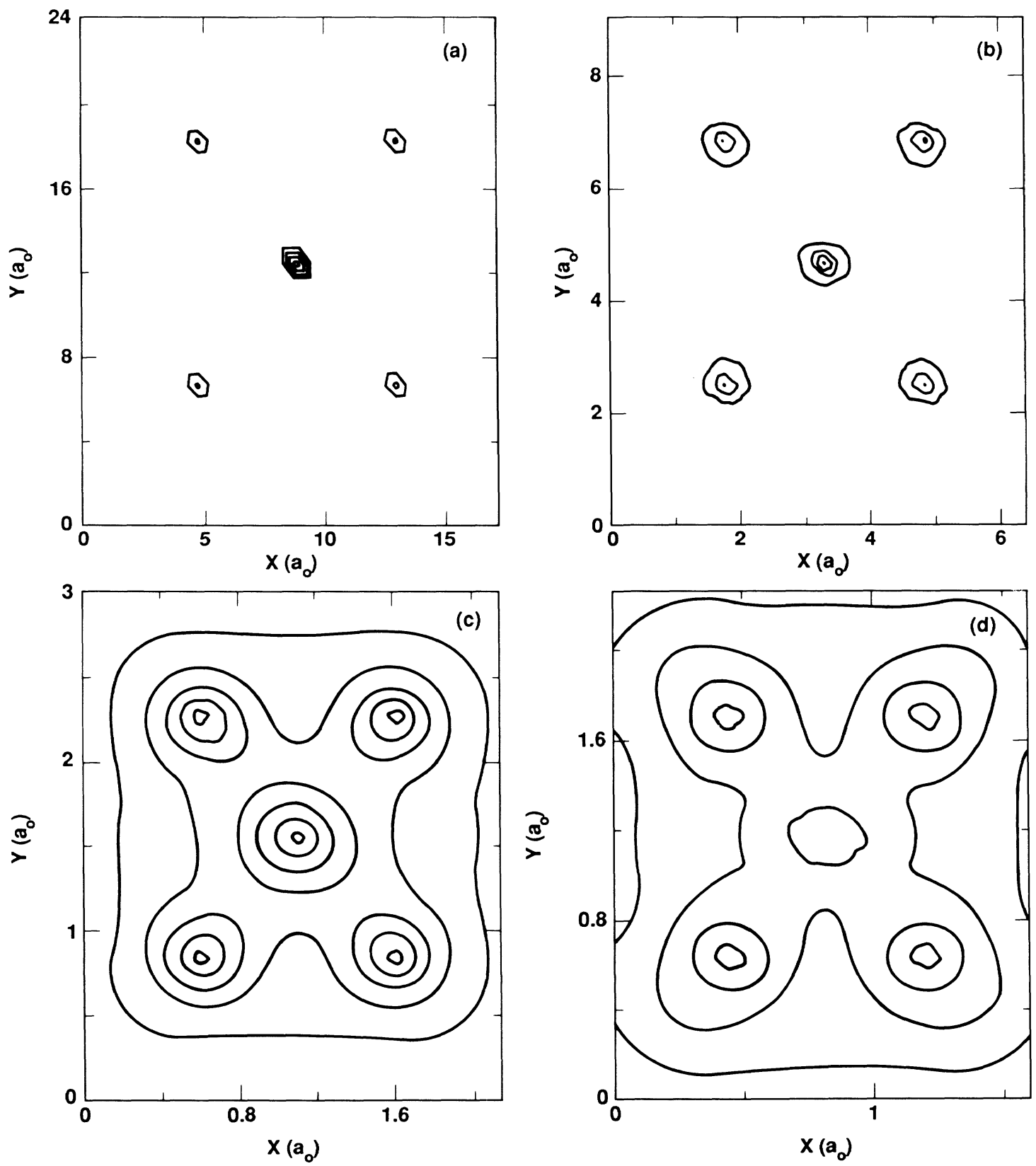

FIG. 3. Contour plots of $d^{3} \rho / e=4 \rho / \rho_{0}$ in a plane containing the central atom and four corner atoms of the cluster. Here $\rho$ is the molecular charge density and $\rho_{0}$ is the average charge density of a body-centered-cubic cluster with lattice spacing $d$. (a) The atomic regime with $d=8 a_{0}$, plotted with a contour interval of 100 . (b) The screened atomic regime at $d=3 a_{0}$ plotted with a contour interval of 10. (c) The quasimolecular regime at $d=a_{0}$ plotted with a contour interval of 1 . There is significant electronic charge density in the internuclear regions. (d) At $d=0.75$ the plasma begins to approach the limit of a homogeneous electron gas. The contour interval here is 1 . 
density plots at four characteristic values of $d$. At low density, the interionic separation is large compared to the spatial extent of bound-state electrons and to the wavelength of the plasma continuum electrons. In this case the plasma is essentially atomic in character. Bound electrons occupy isolated potential wells corresponding to individual nuclei. At higher densities, where the charge clouds of neighboring atoms begin to overlap, the plasma enters the screened atomic regime. Here the nuclear potential wells are still separate, but are close enough for some degree of communication. At densities where the interionic spacing is comparable to the nodal structure of the bound electrons, the quasimolecular regime is encountered in which the electronic charge density is distributed among several nuclei. The potential wells of several nuclei combine to form a single well capable of binding an electron more tightly than an isolated atom. Finally, at very high density, the nuclei are so close together that their associated potential wells are unable to accommodate bound states, and a nearly homogeneous electron gas results. The interionic spacing is small compared to the characteristic wavelength of the electron. Our simple model of a nine-atom cluster begins to break down for $d \leq 0.75 a_{0}$, since the potential used to generate the atomic basis states has too small a negative well to bind an electron. Physically, this means that what bound states do occur will participate in both nearest- and next-nearest-neighbor interactions. We expect that as the high-density limit is approached in a large system, the electronic wave functions will continue to increase in spatial extent, ultimately becoming plane waves confined only by the boundaries of the cluster and perturbed by the nuclear potentials. Although our nineatom cluster is too small to demonstrate this, at $d=0.75 a_{0}$ we begin to see the transition from the quasimolecular to the homogeneous regime.

The modest effect of plasma screening at low and intermediate plasma densities suggests that traditional atomic-based methods for describing bound states will be adequate for many practical applications. At high densities, however, before the limit of a homogeneous plasma is reached, one must approach the plasma as an intrinsically many-body object, which can differ qualitatively from a simple collection of atoms. This observation was made by Collins and Merts ${ }^{2}$ and by Fujima, Watanabe, and Adachi ${ }^{1}$ in their study of zero-temperature compressed matter.

It is interesting that a four-regime description of the metal-insulator transition in expanded $\mathrm{Hg}$ and $\mathrm{Cs}$ was presented some years ago by Cohen and Jortner. ${ }^{5,6}$ They related the smooth transition from insulator to conductor to the formation of extended electronic structures and percolation of the conduction electrons. The present calculations, the first many-atom, finite-electron-temperature study of highly compressed matter, indicate a localization of electrons which is entirely absent from most theories of dense plasma, and suggest a closer relationship between condensed-matter physics and dense plasma physics than may have been heretofore recognized.

In a real plasma we expect the detailed structure of the present model calculation to be obscured by the randomness of the ionic distribution. Bound states will be replaced by bands of levels corresponding to the range of interionic separations found in the plasma. Note, however, that some important physical processes, including shock-wave propagation and electron heat conduction, are fundamentally local in character, in that the important spatial scale is of the order of one electron mean free path. In these cases we expect that the existence of tightly bound quasimolecular orbitals may have an important effect on the plasma dynamics, especially electron energy transport which may be modified by the presence of quasimolecular structures. We are currently in the process of studying the application of the present formalism to the case of energy transport in plasma. In particular, we are adding a molecular-dynamics routine to our computer code which will allow the timedependent motion of the nuclei under the influence of the self-consistently computed electronic charge density.

This work was performed under the auspices of the U.S. Department of Energy by the Lawrence Livermore National Laboratory under Contract No. W-7405ENG-48.

\footnotetext{
(a) Permanent address: Institute for Physical and Chemical Research (RIKEN), Wako, Saitama, Japan.

${ }^{1}$ K. Fujima, T. Watanabe, and H. Adachi, Phys. Rev. A 32, 3585 (1985).

${ }^{2}$ L. A. Collins and A. L. Merts, in Radiative Properties of Hot Dense Matter, edited by J. Davis, C. Hooper, R. Lee, A. Merts, and B. Rozsnyai (World Scientific, Singapore, 1985).

${ }^{3}$ A. Rosen, D. E. Ellis, H. Adachi, and F. W. Averill, J. Chem. Phys. 65, 3629 (1976).

${ }^{4}$ S. M. Younger and A. K. Harrison, in Proceedings of the Eleventh International Conference on Atomic Physics, Paris, 1988 (to be published).

${ }^{5}$ M. H. Cohen and J. Jortner, Phys. Rev. A 10, 978 (1974).

${ }^{6}$ M. H. Cohen and J. Jortner, Phys. Rev. B 15, 1227 (1977).
} 


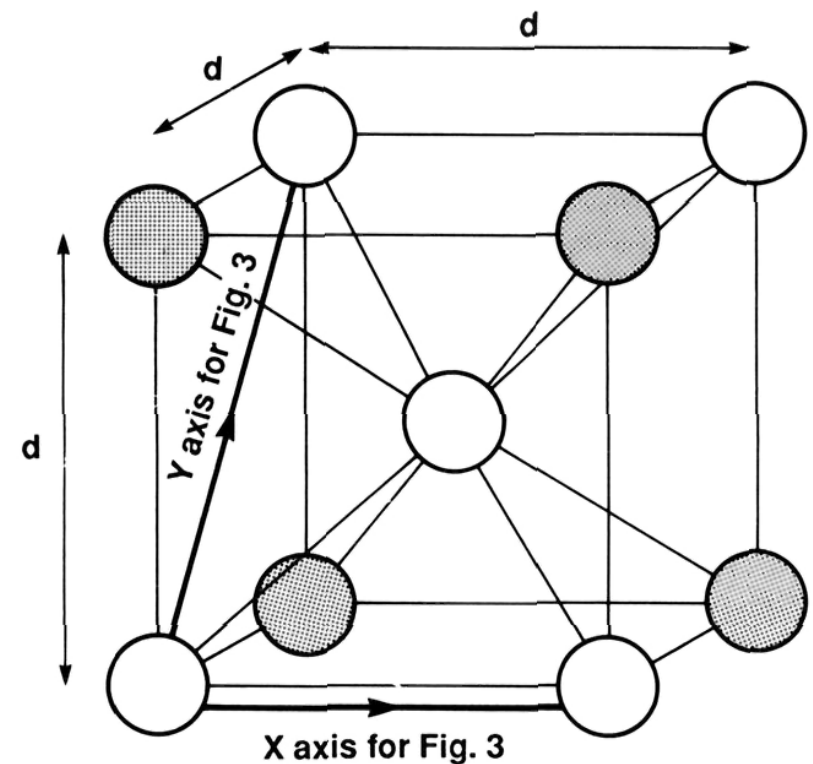

FIG. 1. Geometric configuration of the nine-atom bodycentered-cubic cluster. 


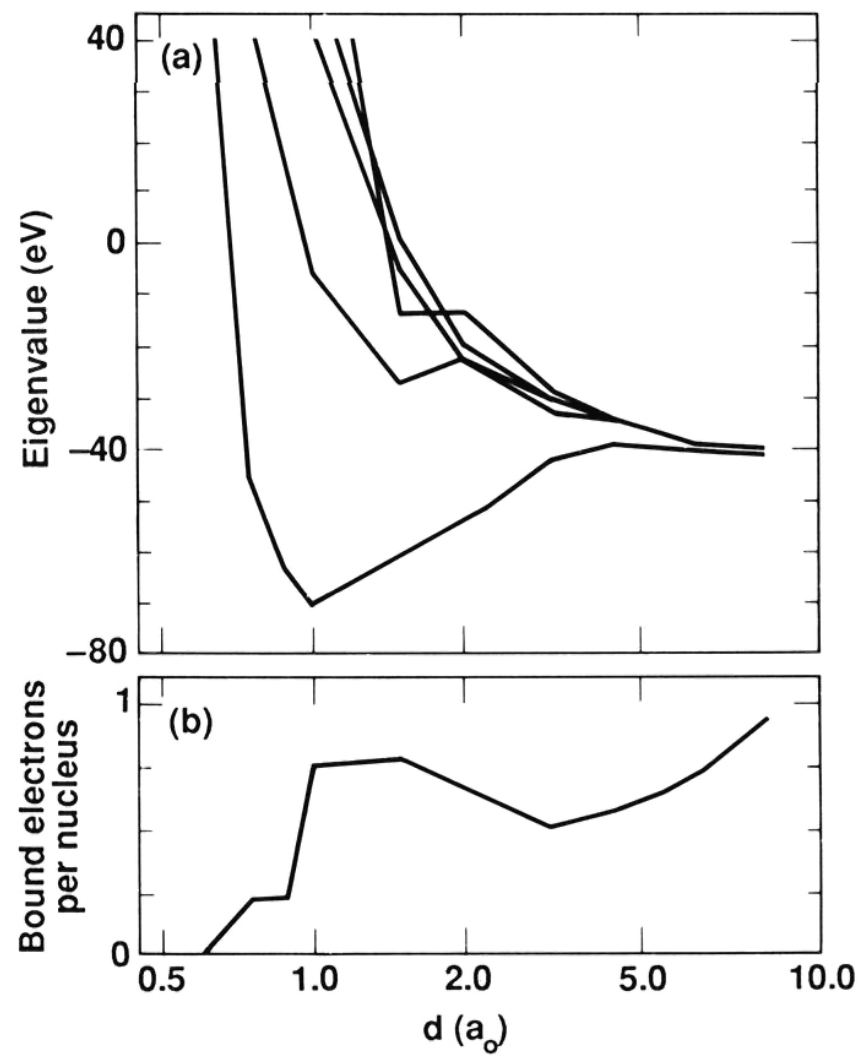

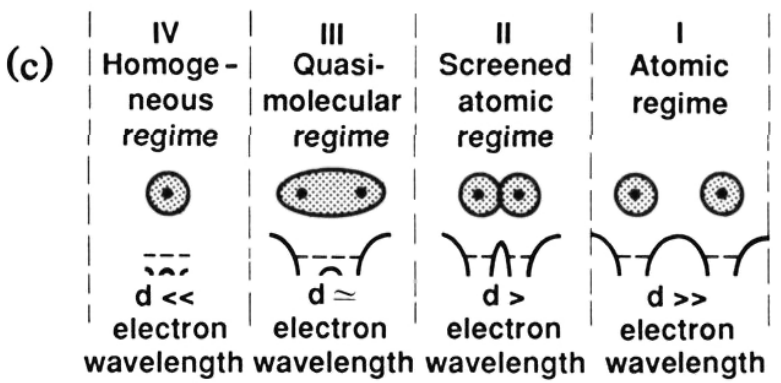

FIG. 2. (a) Eigenvalues of the nine-atom helium cluster which go to the $1 s$ state of atomic helium at large lattice spacing, plotted as a function of the lattice spacing. (b) Average number of bound electrons per atom of the cluster plotted as a function of lattice spacing for a plasma temperature of $50 \mathrm{eV}$. (c) Illustration of the four regimes of the helium plasma in terms of the atomic overlap, the structure of the potential curves determining the electronic motion, and the relationship of the interatomic distance to the characteristic wavelength of the electron. 